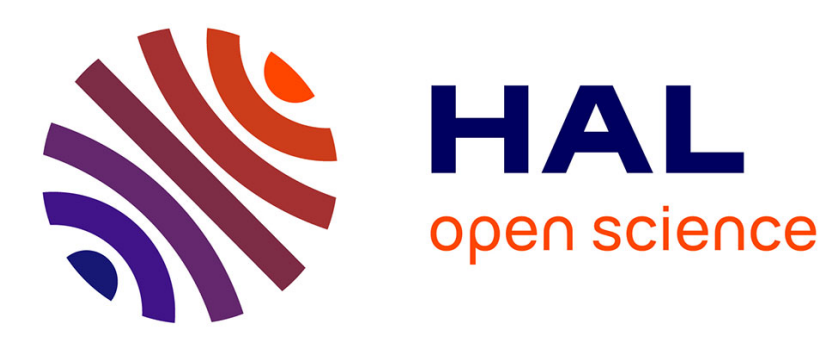

\title{
Carbon/Nitrogen Metabolic Balance: Lessons from Cyanobacteria
}

Cheng-Cai Zhang, Cong-Zhao Zhou, Robert L Burnap, Ling Peng

\section{To cite this version:}

Cheng-Cai Zhang, Cong-Zhao Zhou, Robert L Burnap, Ling Peng. Carbon/Nitrogen Metabolic Balance: Lessons from Cyanobacteria. Trends in Plant Science, 2018, 23 (12), pp.1116 - 1130. 10.1016/j.tplants.2018.09.008 . hal-02992919

\section{HAL Id: hal-02992919 https://hal.science/hal-02992919}

Submitted on 18 Nov 2020

HAL is a multi-disciplinary open access archive for the deposit and dissemination of scientific research documents, whether they are published or not. The documents may come from teaching and research institutions in France or abroad, or from public or private research centers.
L'archive ouverte pluridisciplinaire HAL, est destinée au dépôt et à la diffusion de documents scientifiques de niveau recherche, publiés ou non, émanant des établissements d'enseignement et de recherche français ou étrangers, des laboratoires publics ou privés. 


\section{Highlights}

- Cyanobacteria are simple models among photosynthetic organisms for studying the metabolic regulation of carbon/nitrogen $(\mathrm{C} / \mathrm{N})$, the two most abundant nutrient elements for all living organisms.

- 2-oxoglutarate (2-OG), an intermediate from the Krebs cycle, serves as a carbon skeleton for nitrogen assimilation and as a signal of nitrogen limitation, whereas 2phosphoglycolate (2-PG), an intermediate from photorespiration, acts as a signal of inorganic carbon limitation.

- The levels of 2-PG and 2-OG are inversely correlated, and their ratio reflects the $\mathrm{C} / \mathrm{N}$ metabolic balance.

- The transcriptional activator NtcA is a sensor of 2-OG, and the transcriptional repressor $\mathrm{NdhR}$ is a sensor of both 2-OG and 2-PG. These regulators together balance $\mathrm{C} / \mathrm{N}$ metabolic networks by switching on or off the expression of genes involved mainly in the uptake and assimilation of carbon and nitrogen sources.

- The signalling role of 2-OG and 2-PG in $\mathrm{C} / \mathrm{N}$ balance is likely conserved in other photosynthetic organisms. 


\section{Carbon/nitrogen metabolic balance: lessons from cyanobacteria}

3

4

5 Cheng-Cai Zhang ${ }^{1,5^{*}}$, Cong-Zhao Zhou ${ }^{2}$, Robert L. Burnap ${ }^{3}$ and Ling Peng ${ }^{4}$

6

7

8

${ }^{1}$ Key Laboratory of Algal Biology, Institute of Hydrobiology, the Chinese Academy of

9 Sciences, Wuhan, Hubei 430072, People's Republic of China

10

${ }^{2}$ School of Life Sciences and Hefei National Laboratory for Physical Sciences at the

Microscale, University of Science and Technology of China, Hefei, Anhui, 230027, People's

Republic of China

${ }^{3}$ Department of Microbiology and Molecular Genetics, Henry Bellmon Research Center,

Oklahoma State University, Stillwater, OK 74078, USA

${ }^{4}$ Aix-Marseille Université, CNRS, Centre Interdisciplinaire de Nanoscience de Marseille,

CINaM UMR 7325, «Equipe Labellisée Ligue Contre le Cancer», 13288 Marseille, France

CINaM UMR 7325, 13288 Marseille, France

${ }^{5}$ Aix Marseille Univ, CNRS, LCB, Marseille, France

*Corresponding author: Zhang, C.-C. (cczhang@ihb.ac.cn).

Keywords: nitrogen metabolism, carbon fixation, photorespiration, signal transduction 30 
32 Abstract

33 Carbon and nitrogen are the two most abundant nutrient elements for all living organisms, 34 and their metabolism is tightly coupled. What are the signalling mechanisms that cells use to 35 sense and control the carbon/nitrogen $(\mathrm{C} / \mathrm{N})$ metabolic balance following environmental changes? Based on studies in cyanobacteria, it was found that 2-phosphoglycolate derived

37 from photorespiration and 2-oxoglutarate from the Krebs cycle act respectively as the carbonand nitrogen-starvation signals, and their concentration ratio likely reflects the status of the $\mathrm{C} / \mathrm{N}$ metabolic balance. We will present and discuss the regulatory principles underlying the

40 signalling mechanisms, which are likely to be conserved in other photosynthetic organisms.

41 These concepts may also contribute to developments in the field of biofuel engineering or 42 improvements in crop productivity.

43

44

45 
47 Carbon and nitrogen metabolism is essential for every biological system, since all major cellular components, including genetic materials, proteins, pigments, energy carrier molecules, etc., are derived from these activities. Carbon and nitrogen metabolism is tightly coupled in different living organisms. In prokaryotes and plants, such a coupling mechanism can be attributed to two major factors. First, the two elements are the most abundant in cells intensifying the requirement for coordination mechanisms to avoid metabolic inefficiencies; second, nitrogen assimilation depends on the availability of a carbon skeleton for biosynthesis, and consequently the limitation or oversupply of one element strongly affects the metabolism of the other. Therefore, the $\mathrm{C} / \mathrm{N}$ stoichiometry in different organisms varies within a relatively narrow range; for example, the mass ratio is $\mathrm{C} / \mathrm{N}=31 / 4$ in phytoplankton [1].

Because properly balanced metabolism of carbon and nitrogen is necessary for optimal growth, different levels of regulation exist in cells in order to control the uptake and assimilation of various nitrogen and carbon sources whose supply may vary under different environmental conditions [2-4]. Such regulation may occur at various levels of control ranging from the allosteric modulation of the activity of nutrient assimilation proteins to a variety of mechanisms controlling the expression of genes encoding these structural proteins. The latter includes a rich variety of transcriptional and post-transcriptional mechanisms, some of which are still being experimentally clarified [5-7].

Whatever the type of regulation involved, two major questions arise: how do cells sense the metabolic status, and what signal transduction mechanisms do cells use to maintain a proper balance of nitrogen and carbon metabolism? Recently, tremendous progress has been made towards answering these questions by using cyanobacteria as a model for photosynthetic organisms, and this review serves to summarize the data and highlight the mechanisms underlying the signaling and transcriptional regulation involved in carbon and nitrogen metabolic control. We will also discuss the concept for controlling $\mathrm{C} / \mathrm{N}$ metabolic balance. Cyanobacteria are the ancestors of plastids [8]; therefore, cyanobacteria and plants may share common features, especially in terms of the signaling mechanisms, for balancing $\mathrm{C} / \mathrm{N}$ metabolism.

\section{Coupling between carbon and nitrogen metabolic pathways}

To facilitate understanding, we briefly summarize here the metabolic coupling of nitrogen and 
carbon assimilation in cyanobacteria (Fig. 1). As for other autotrophic photosynthetic organisms, $\mathrm{CO}_{2}$ is the primary carbon source for cyanobacteria, although some cyanobacterial strains can also use a sugar or other organic carbon as the carbon source $[4,9,10]$. However, as with eukaryotic algae, the availability of $\mathrm{CO}_{2}$ is often growth-limiting because of specific physical factors such as the low diffusion rates in the aqueous environment. Moreover, dissolved $\mathrm{CO}_{2}$ chemically speciates into several forms of inorganic carbon according to the general reaction describing the inorganic carbon pool:

$$
\mathrm{CO}_{2}+\mathrm{H}_{2} \mathrm{O} \rightleftharpoons \mathrm{H}_{2} \mathrm{CO}_{3} \rightleftharpoons \mathrm{HCO}_{3}^{-}+\mathrm{H}^{+} \rightleftharpoons \mathrm{CO}_{3}^{2-}+2 \mathrm{H}^{+}
$$

Note that the equilibrium of the reaction is strongly $\mathrm{pH}$ dependent such that the fraction of the total inorganic pool existing as $\mathrm{CO}_{2}$ is favored by acidic conditions, whereas bicarbonate $\mathrm{HCO}_{3}{ }^{-}$dominates under neutral and alkaline conditions. $\mathrm{CO}_{3}{ }^{2-}$ can be ignored in general as it exists only in strong alkaline condition, which is seldom the case within normal cells. Cyanobacteria and algae are typically capable of acquiring either $\mathrm{CO}_{2}$ or $\mathrm{HCO}_{3}^{-}$. Because both forms may be acquired and are rapidly interchanged within the cell, the symbol $C_{i}$ is often utilized to refer to total inorganic carbon (i.e. $\mathrm{C}_{\mathrm{i}}=\mathrm{CO}_{2}+\mathrm{HCO}_{3}{ }^{-}$) (Fig. 1). $\mathrm{CO}_{2}$ fixation is catalyzed by Rubisco in the Calvin-Benson-Bassham (CBB) cycle. The reaction involves the carboxylation, using $\mathrm{CO}_{2}$ and ribulose 1,5-biphosphate (RuBP), yielding two molecules of the three carbon 3phosphoglycerate (3-PGA) [11-13]. However, Rubisco has a notoriously low affinity for $\mathrm{CO}_{2}$ $(\mathrm{Km}>150 \mu \mathrm{M})$ and, problematically, the enzyme also catalyzes the competing and wasteful oxygenase reaction between $\mathrm{RuBP}$ and $\mathrm{O}_{2}$, which yields one molecule of the two-carbon 2Phosphoglycolate (2-PG) and one 3-PGA rather the two 3-PGA molecules of the carboxylation reaction. The oxygenase reaction has resulted in the evolution of scavenging photorespiratory pathways, which recover and recycle the carbon that would otherwise be lost $[11,13]$. As a consequence of these factors, carbon fixation in cyanobacteria critically depends upon the socalled $\mathrm{CO}_{2}$-concentrating mechanism $(\mathrm{CCM})[4,14,15]$. Cyanobacteria actively acquire $\mathrm{C}_{\mathrm{i}}$, either as $\mathrm{HCO}_{3}^{-}$or as $\mathrm{CO}_{2}$, using a complex set of transporters and uptake enzymes. Bicarbonate uptake involves active membrane transporters, such as the sodium-dependent bicarbonate transporter, SbtA or BicA. Energized $\mathrm{CO}_{2}$ uptake occurs via the enzymatic hydration of $\mathrm{CO}_{2}$ catalyzed by specialized forms of the NDH-1 complex located in thylakoid membrane. These complexes use redox energy to drive the conversion of $\mathrm{CO}_{2}$ to $\mathrm{HCO}_{3}{ }^{-}$in the cytoplasm and simultaneously draw in $\mathrm{CO}_{2}$ from the environment. Accordingly, these are not simple carbonic anhydrases, but enzymes capable of using metabolic energy to create a 
disequilibrium in favor of $\mathrm{HCO}_{3}{ }^{-}$accumulation. Together, these systems produce and maintain high concentrations of $\mathrm{HCO}_{3}{ }^{-}$in the cytoplasm to supply the carboxysome, which encloses the entire cellular complement of Rubisco within a protein shell. The carboxysome is the first 113 identified member of a large class of prokaryotic structures called bacterial microcompartments, which generally partition specific metabolic reactions within the cytoplasm of the bacterial species that possess them. In the case of the carboxysome, RuBP and $\mathrm{HCO}_{3}{ }^{-}$are imported through specific pores in the protein shell and 3-PGA is exported through pores in the shell to supply the CBB cycle, although the details of these transport process are only beginning to emerge. Similarly, the oxygenase reaction product, 2-PG, must also be able to diffuse out of the carboxysome, although again, the precise mechanisms for passage through the protein shell of the carboxysome remain to be elucidated [14]. Importantly, the carboxysome also contains a carbonic anhydrase with functions to convert

122 imported $\mathrm{HCO}_{3}$ into $\mathrm{CO}_{2}$ in the proximity of Rubisco. Therefore, given an abundant supply of 123 $\mathrm{HCO}_{3}{ }^{-}$, the active sites of Rubisco with the carboxysome are saturated with $\mathrm{CO}_{2}$, which enables

124 high rates of $\mathrm{CO}_{2}$ fixation. This enormously diminishes the competing reaction with $\mathrm{O}_{2}$; however under conditions of $\mathrm{C}_{\mathrm{i}}$ limitation or in mutants that fail to accumulate sufficient $\mathrm{HCO}_{3}{ }^{-}$ in the cytoplasm, significant rates of the oxygenase reaction have been observed to occur $127[11,13]$. Thus, the CCM relies on the ability of the $C_{i}$-uptake systems to concentrate high concentrations of $\mathrm{HCO}_{3}{ }^{-}$within the cytoplasm in order to create a steep concentration gradient that promotes rapid net diffusion of $\mathrm{HCO}_{3}{ }^{-}$into the carboxysome. A substantial proportion of 3-PGA, produced by the CBB cycle, is converted to acetyl-CoA which feeds the essential central metabolic pathway, the Krebs cycle (also known as the citric acid cycle, tricarboxylic acid cycle or TCA cycle), thus leading to the production of intermediates for biosynthesis $[4,13,16,17]$ (Fig. 1). As discussed below, the coordination of $\mathrm{N}$ - and Cmetabolism depends, in part upon cytoplasmic concentrations of several metabolites affected by the efficiency of the $\mathrm{CO}_{2}$-fixation reactions.

Cyanobacteria can use different inorganic nitrogen sources, mostly in the form of nitrate or ammonium. Many cyanobacterial strains can synthesize the enzyme nitrogenase for $138 \mathrm{~N}_{2}$ fixation $[3,9]$. Whatever the form of inorganic nitrogen that is taken up into the cells, it is 139 always converted into ammonium, the least costly nitrogen form for assimilation (Fig. 1). 140 Nitrate requires two additional enzymes, nitrate reductase and nitrite reductase and reduced 141 cofactors, in order to be reduced to ammonium, whereas $N_{2}$ fixation is even more 
energetically expensive requiring both reduced cofactors and up to 16 ATP molecules per ammonium. Moreover, it depends on the nitrogenase complex which needs to operate under

144 micro-oxic conditions, thereby requiring special adaptations. Some filamentous 145 cyanobacteria, such as Anabaena/Nostoc PCC 7120 can form heterocysts, intercalated among 146 vegetative cells, to provide a micro-oxic intracellular environment for $\mathrm{N}_{2}$ fixation or, 147 alternatively, $\mathrm{N}_{2}$ fixation and photosynthetic $\mathrm{O}_{2}$ evolution occur in the same cell, but the 148 activities are diurnally regulated using a circadian clock so that $\mathrm{N}_{2}$ fixation is restricted to the 149 night [3,18-20]. Hierarchically, ammonium represses the use of alternative nitrogen sources, 150 and heterocyst differentiation and $\mathrm{N}_{2}$ fixation take place only when neither ammonium nor 151 nitrate is available in the growth medium.

Two ammonium-assimilation pathways are known, the glutamine synthetase153 glutamate synthase (GS-GOGAT) cycle, and direct assimilation through the reaction catalyzed 154 by glutamate dehydrogenase $(\mathrm{GDH})$. Both pathways require the carbon skeleton 2155 oxoglutarate (2-OG, also known as $\alpha$-ketoglutarate), one of the intermediates of the Krebs 156 cycle [21,22] (Fig. 1). Therefore, ammonium assimilation using 2-OG provides the metabolic 157 basis for coupling between nitrogen and carbon metabolism. In the GS-GOGAT cycle, GS 158 assimilates ammonium using glutamate and ATP to synthesize glutamine, while GOGAT catalyzes glutamate formation using glutamine, 2-OG and NADPH. The second route for ammonium assimilation, catalyzed by GDH using 2-OG, does not play a major role in cyanobacteria, and the majority of cyanobacterial species whose genomes have been sequenced do not even contain the gene encoding GDH [21,23]. Thus, the GS-GOGAT cycle constitutes the major pathway for ammonium assimilation in cyanobacteria, and hence the central crossroad for carbon and nitrogen metabolism (Fig. 1). Glutamine and glutamate are also the major intracellular amino group donors for the synthesis of several other amino acids, and for purine and pyrimidine nucleobases, as well as other nitrogen-containing compounds $167[24,25]$.

Both carbon and nitrogen assimilation are under multiple control mechanisms depending on the availability or the nature of the nutrients in the environment. To understand 170 how cyanobacteria regulate nitrogen and carbon metabolism, we will first focus on the nature 171 of the signals that allow cells to sense the intracellular metabolic status when nitrogen and 172 carbon metabolism becomes unbalanced, and then we will review the signaling mechanisms 173 that allow cells to regain their metabolic balance in order to better adapt to environmental 
changes. While the focus of this analysis is based upon the transcriptional regulation, it is important to note that researchers performing global transcriptome and proteome analyses

176 have observed significant differences between fold-changes in transcript and the corresponding protein levels in cyanobacteria $[26,27]$. This indicates the existence of posttranscriptional factors in the control of gene expression in cyanobacteria. However, it is also important to note that there is generally a good qualitative correspondence between the levels of transcripts and proteins, with the latter typically exhibiting more muted fold changes in response to environmental changes.

182

\section{The nitrogen-starvation signal 2-OG and its receptors}

It has been postulated for a while that 2-OG acts as a signal in the control of nitrogen metabolism because of its role as a carbon skeleton in nitrogen assimilation and because its levels can vary according to nitrogen availability [28,29] (Fig. 1). Indeed, in both the filamentous heterocyst-forming diazotrophic cyanobacterium Anabaena PCC 7120 and the unicellular non-diazotrophic strain Synechocyctis PCC 6803, 2-OG accumulates rapidly upon limitation of nitrogen supply in the growth medium $[28,29]$. A signaling function for 2-OG has also been proposed in a variety of biological systems, including bacteria, animals and humans $[2,25,30-35]$. In proteobacteria, for example, $2-O G$ is involved in the control of metabolism $[25,36]$, although the signaling mechanism is in many ways different from that in cyanobacteria, as will be discussed later in this review.

Because 2-OG is a critical metabolite rapidly incorporated into a variety of $\mathrm{N}$-containing compounds, one crucial question is how to experimentally discriminate its metabolic function from its signaling function, and how its direct effects are distinguished from the indirect effects of its metabolic derivatives. One way to tackle this problem is through the use of nonmetabolizable analogs, in which a stable bioisostere function replaces the keto group in 2OG, the site of amidation in ammonium assimilation in the GS-GOGAT cycle. Specifically, several nonmetabolizable analogs of 2-OG were chemically synthesized and tested in Anabaena PCC 7120 [29,37-40]. This organism was chosen for these tests because it has, under nitrogen-limited conditions, a phenotype (formation of $\mathrm{N}_{2}$-fixing heterocysts) which is easily observable under a light microscope $[3,18]$. Among the synthesized nonmetabolizable analogs of 2-OG, 2,2-difluoropentanedioic acid (DFPA) is particularly interesting (Fig. 2). DFPA 
nonmetabolizable fluorinated methylene entity [29]. Since fluorine is in general absent in biological systems and cells, fluorinated compounds can be readily tracked in intact cells using

${ }^{19} \mathrm{~F}-\mathrm{NMR}$, with little or no background interference [41,42]. Accumulation of DFPA in filaments of Anabaena PCC 7120 acts as a trigger for heterocyst differentiation, even when ammonium, which normally represses heterocyst differentiation, is present in the growth medium. DFPA can be recognized by both a 2-OG permease, KgtP, and a 2-OG receptor, NtcA $[29,43]$. These results provided the first in vivo evidence for a direct role of 2-OG as a nitrogen starvation signal in cyanobacteria.

Two 2-OG receptors have been known for a while in cyanobacteria, NtcA and PII $[2,3]$. The third, NdhR, was more recently established as a 2-OG receptor [44-46]. Here we will first focus on NtcA and PII, because NdhR is also a receptor of 2-PG [46-48], and will be discussed later in this review as a key element in $\mathrm{C} / \mathrm{N}$ metabolic balance.

$\mathrm{PII}$ is a trimeric regulatory protein which is highly conserved in both bacteria and plants $[2,35,49]$. PII exerts its function mainly through protein-protein interactions mediated by the flexible and outward-extended T-loop present on each monomer. The binding of 2-OG determines the conformation of the T-loop, and hence the interaction with the partners in nitrogen metabolism [50]. Among the interacting partners of PII are PipX, which is involved in NtcA-mediated transcriptional control by acting as a co-activator of NtcA, and NAGK (N-acetylL-glutamate kinase), a key enzyme for arginine synthesis [51,52]. The interaction of PII with either PipX or NAGK occurs under nitrogen sufficiency, and thus when the intracellular 2-OG level is low [51-54]. PII relieves the feedback inhibition of Arg on the activity of NAGK, resulting in an increase of Arg for cyanophycin synthesis as a nitrogen reserve as a consequence of nitrogen sufficiency $[52,53]$; at the same time, PII sequesters PipX, preventing its role as a coactivator of NtcA [51,54]. Several reviews dealing with the detailed mechanism of PII signaling are available for readers interested in more details in this topic $[2,35,49]$. Importantly, additional proteins in the PII-family are recently reported as being involved in carbon assimilation $[7,55]$. The small PII-type protein StbB is physically linked and co-expressed with the bicarbonate uptake transporter, StbA. Structural and physiological analyses indicate that this protein is involved in CAMP sensing and responds to cyanobacterial inorganic carbon status. This indicates the existence of a direct regulatory connection between inorganic carbon availability and broader carbon metabolism. These findings resonate with the fact that 
in carbon catabolite repression and the CAMP levels are themselves regulated by 2-OG. While it remains to be seen whether this also applies to cyanobacteria, it points to the possibility that new modes of regulation affecting $\mathrm{C} / \mathrm{N}$ balance may still be found.

NtcA is a dimeric global transcriptional factor belonging to the CRP/CAP family [3]. $\mathrm{NtcA}$ activates the expression of genes required for heterocyst development in Anabaena PCC 7120 and the use of alternative nitrogen sources under nitrogen deprivation. Recent structural studies of NtcA from both Anabaena PCC 7120 and Synechocystis PCC 6803 provide further insight into the molecular mechanism of 2-OG signaling mediated through NtcA $[43,56]$. Specifically, each NtcA monomer harbors a 2-OG binding pocket, and 2-OG binding generates two allosteric effects in NtcA: first, there is an increase in the contact area between the two monomers through the two long $\mathrm{C}$-helices, and then this change propagates towards the DNAbinding domain by shortening the distance between the two helices responsible for DNA binding (Fig. 2). In the native or apo form of NtcA, the distance between the two DNA-binding helices is about $37 \AA$, whereas in the 2-OG-bound form, it becomes $34 \AA$, which corresponds to the distance between successive DNA major grooves [43]. Furthermore, transcriptional activity assays in vitro also reveal that interaction of 2-OG-free NtcA with its target promoters is not sufficient for activation of gene transcription, and 2-OG binding to NtcA is necessary to initiate the process of transcriptional control [57].

Based on all information available so far, a molecular model of the signaling mechanism in response to nitrogen starvation can be proposed. Upon nitrogen deprivation, 2-OG accumulates rapidly in cells; it binds to PII and regulates nitrogen metabolism by proteinprotein interactions on the one hand, and it interacts with NtcA which activates genes involved in the uptake and assimilation of alternative nitrogen sources on the other (Fig. 2). For those cyanobacteria able to form heterocysts, such as Anabaena PCC 7120, 2-OG also serves as a trigger for the initiation of heterocysts, which fix atmospheric $N_{2}$ using nitrogenase $[29,58]$.

Evidence for 2-PG as a signal of carbon starvation

$\mathrm{CO}_{2}$ assimilation is catalyzed by Rubisco, the most abundant enzyme on earth. As mentioned above, $\mathrm{CO}_{2}$ and $\mathrm{O}_{2}$ are competitive substrates for the reaction with RuBP in the active site of Rubisco [11-13] (Fig. 3). Through the carboxylase activity of Rubisco, $\mathrm{CO}_{2}$ is combined with RuBP to produce 3-PGA; The remainder of the CBB cycles serves to regenerate RuBP and 
back to RuBP through the CBB pathway, while the rest of the 3-PGA is used to make sugar to

271 feed carbon metabolism and the Krebs cycle (Fig. 3). Through the oxygenase activity of 272 Rubisco, each RuBP molecule is converted to 1 molecule of 2-PG and 1 molecule of 3-PGA. 2273 PG, being toxic, is then metabolized by the so-called photorespiration pathway $[11-13,59]$. 274 Thus, the activity of Rubisco is dependent on the relative ratio of $\mathrm{CO}_{2}$ and $\mathrm{O}_{2}$.

275 Two transcriptional regulators have been extensively studied in cyanobacteria for the 276 control of genes involved in carbon acquisition and/or metabolism: NdhR (CcmR) and CmpR $277[45,60]$. A third one, an AbrB-type regulator, is also involved in the control of gene expression 278 according to carbon availability in Synechocystis PCC 6803, but the underlying mechanism 279 remains largely unknown [61]; the same is true for PacR, a LysR-type transcriptional regulator 280 identified in Anabaena PCC 7120 [62]. Both NdhR and CmpR are LysR-type transcription 281 factors, and their function in cyanobacteria has been well documented [4]. Briefly, CmpR is an 282 activator of the $\mathrm{cmp}$ operon; this operon encodes the high-affinity bicarbonate transporter 283 BCT1, which is induced under low- $\mathrm{CO}_{2}$ conditions [60,63] (Fig. 3). NdhR is a repressor, whose 284 target genes, among others, encode several $\mathrm{CO}_{2}$ or bicarbonate uptake systems located on 285 either the cytoplasmic ( $\left.\mathrm{Na}^{+}-\mathrm{NDH}-1, \mathrm{Sbt}\right)$ or the thylakoid membranes $\left(\mathrm{Ndh}-1_{3}\right)[4,44-$ 286 46,48,64,65] (Fig. 3). During carbon sufficiency, genes encoding CCM-related functions are therefore repressed by NdhR. LysR-type transcription factors are usually under allosteric control; thus, the existence of effectors that interact with either CmpR or NdhR was expected. Metabolites from photorespiration or CBB, whose levels could oscillate according to carbon availability, have been tested in various studies $[45,47,48,63,64,66]$. DNA gel-shift assays indicate that RuBP and 2-PG can both bind to $\mathrm{CmpR}$ to activate the $\mathrm{cmp}$ operon and enhance $\mathrm{CO}_{2}$ uptake under carbon-limiting conditions $[48,63]$. However, the effect of 2-PG on the transcription of the $\mathrm{cmp}$ operon mediated by $\mathrm{CmpR}[47,66]$ will need to be confirmed in vivo, and the effect of both RuBP and 2-PG on $\mathrm{CmpR}$ has yet to be demonstrated from structural studies. In contrast, much progress has been made in identifying the effectors of NdhR, which is considered as the most critical regulator of cyanobacterial CCM [4]. Using surface plasmon resonance, it was shown that 2-OG and $\mathrm{NADP}^{+}$could both interact with NdhR and enhance its DNA binding activity [48]. In a more recent study, 4 metabolites, 2-OG, 2-PG, RuBP, and NADP+, were investigated, among which 2-OG and 2-PG were found to interact with NdhR: 2OG enhances the DNA-binding activity, and is therefore a co-repressor, while 2-PG prevents NdhR from DNA binding, thus acting as an anti-repressor or inducer [46]. These observations 
were further confirmed in vivo by following the effects on gene expression after changes in the 2-OG or 2-PG levels. Indeed, increasing the cellular concentration of 2-OG by adding the membrane-permeable precursor, dimethyl 2-oxoglutarate (dmOG) (Fig. 2a), enhanced the repressive effect of NdhR [46], whereas decreasing the 2-PG pool in the cells by overexpressing a gene encoding phosphoglycolate phosphatase brought down the expression levels of the NdhR regulon $[46,47]$.

Crystal structure analyses and structural simulation have provided a mechanism whereby 2-PG and 2-OG effectors control the activity of the NdhR repressor [46] (Fig. 3b). $\mathrm{NdhR}$ is a tetramer composed of two compact subunits and two extended subunits. Each subunit contains a DNA-binding domain (DBD) and a regulatory domain (RD), bridged by a long $\alpha$-helical linker. The NdhR tetramer contains two 2-OG binding pockets formed at the interface between two RD domains. In the 2-OG/NdhR complex, the distance between the DNArecognition helices in the two neighboring DBDs is $29 \AA$, which matches a continuous DNA major groove and is thus favorable for DNA binding. Therefore, 2-OG is a co-repressor that keeps NdhR bound to its DNA targets, providing a rationale for the effect of 2-OG on the repression of the NdhR regulon. 2-PG binds to NdhR at the intradomain cleft of each RD pair, and the distance between the two DNA-binding helices becomes $25 \AA$, which is too short to fit the DNA major groove. Thus, 2-PG acts as an anti-repressor or inducer of the NdhR regulon. Although 2-PG and 2-OG bind to different sites in NdhR with a similar affinity, their binding is mutually exclusive because of the structural incompatibility between the two-bound forms $322[46]$.

All the data summarized above, together with the fact that the 2-PG level transiently increases in cells upon carbon limitation [44], demonstrate that 2-PG acts as a carbon starvation signal, as already suggested by the effect of reduced 2-PG on the expression of genes related to CCM [47]. Furthermore, NdhR is another 2-OG receptor in addition to PII and 327 NtcA in cyanobacteria.

\section{$\mathrm{C} / \mathrm{N}$ metabolic balance through the integration of 2-OG and 2-PG signals}

Based on all the studies using different approaches, a model can be proposed to describe how cyanobacteria achieve $\mathrm{C} / \mathrm{N}$ metabolic balance by transcriptional regulation (Fig. 4). Most of the data have been derived using three model cyanobacterial strains, namely Synechococcus elongatus PCC 7942, Synechocystis PCC 6803, and Anabaena PCC 7120. 

and nitrogen metabolism. Cyanobacteria in general lack 2-OG dehydrogenase, thus have an incomplete Krebs cycle $[16,17,67]$; in such a case, $2-O G$ can be considered as an end-product of this metabolic pathway (Fig. 1). The intracellular pool of 2-OG, derived from the Krebs cycle, accumulates transiently upon, and serves as a signal for, nitrogen deprivation $[28,29]$. This is consistent with its role as a carbon skeleton for nitrogen assimilation through the GS-GOGAT cycle (Fig. 1). The level of 2-PG, a metabolite of photorespiration, increases transiently in cells following carbon limitation [44,47], and serves as a carbon starvation signal, which is directly linked to the activity of Rubisco (Fig. 3). Indeed, when the level of $\mathrm{CO}_{2}$ is high, the higher $\mathrm{CO}_{2} / \mathrm{O}_{2}$ ratio favors the carboxylase activity of Rubisco, and thus the production of 3-PGA; in contrast, when $\mathrm{CO}_{2}$ becomes limiting, the lower ratio of $\mathrm{CO}_{2} / \mathrm{O}_{2}$ favors the oxygenase activity of Rubisco, leading to the production of 2-PG. Because of the strong coupling between carbon and nitrogen metabolism, especially through the GS-GOGAT cycle (Fig. 1), nitrogen limitation can also be considered as carbon oversupply, and nitrogen oversupply also correlates with carbon limitation in cells. Altogether, our current knowledge suggests that the levels of 2-OG and 2-PG are somehow inversely correlated in cells, and their ratio may reflect the carbon and nitrogen metabolic status in cyanobacteria. The transient increase in 2-OG or 2-PG observed under a particular condition is characteristic of many signaling molecules, such as $\mathrm{Ca}^{2+}, \mathrm{cAMP}$, etc. $[68,69]$.

Secondly, combining with previously published proposal and hypothesis with recent data based on genetic, biochemical and structural studies $[4,28,29,43,45-47,56,64,70]$, we suggest a model for the transcriptional control of $\mathrm{C} / \mathrm{N}$ balance, which is illustrated in Fig. 4. When $\mathrm{C} / \mathrm{N}$ metabolism is unbalanced because of nitrogen limitation (or carbon oversupply) (Fig. 4a), the 2-OG pool increases while the 2-PG pool decreases for the reasons explained above. Consequently, 2-OG binds to both NtcA and NdhR. The 2-OG/NtcA complex upregulates genes involved in the uptake of alternative nitrogen sources such as nitrate, or heterocyst differentiation and $\mathrm{N}_{2}$ fixation for diazotrophic heterocyst-forming strains such as Anabaena PCC $7120[3,18]$; meanwhile, the 2-OG/NdhR complex downregulates CCM so that bicarbonate uptake goes down. PII also forms a complex with 2-OG and regulates nitrogen assimilation accordingly through protein-protein interaction [2]. Together, the increased uptake and assimilation of nitrogen sources and the decreased uptake of inorganic carbon 
366 (or nitrogen oversupply), Rubisco switches towards its oxygenation activity, leading to 367 accumulation of a 2-PG pool while 2-OG levels decrease because less carbon will go to feed the Krebs cycle (Fig. 3, Fig. 4b). In such a case, NtcA exists in its apo form, which is unfavorable for DNA binding and activation of genes involved in the uptake and assimilation of nitrogen sources; at the same time, 2-PG forms a complex with NdhR, making it unable to bind to DNA, which relieves the repression of genes involved in carbon acquisition. Thus, decreased uptake and assimilation of nitrogen, alongside increased uptake of inorganic carbon, helps cells to rebalance their carbon and nitrogen metabolism.

Some aspects of this model still require further experimental studies. For example, the model assumes concomitant changes in the 2-OG and 2-PG pools upon changes in the availability of carbon or nitrogen sources, consistent with the fact that 2-OG and 2-PG cannot bind to the same molecule of NdhR at the same time [46]. Although transient changes in the pools of these two metabolites have been observed $[28,29,44,47]$, it remains unknown to what extent and on what time-scale their changes are correlated, and the current data are sometimes even contradictory [4]. It will also be relevant to identify the input of other potential signals and regulators involved in $\mathrm{C} / \mathrm{N}$ metabolism, as well as their interaction with the 2-OG and 2-PG signaling pathways. For example, could RuBP or 3-PGA act as effectors during carbon metabolism as suggested by some studies [4]?

The 2-OG and 2-PG signals control the uptake of nitrogen and carbon sources or the initiation step of heterocyst differentiation when $\mathrm{N}_{2}$ fixation is needed, and 2-OG also extensively regulates later steps in nitrogen and carbon metabolism through PII and NtcA. Indeed, NtcA is not just required for nitrogen control, it is also a global regulator directly involved in the modulation of carbon metabolism, photosynthesis, and oxidative stress [3]. For example, $c m p R$ is possibly a target of NtcA in Anabaena PCC 7120 [71], and ccmK2 encoding a CCM-function protein was also identified by ChIP-seq as a direct target of NtcA in Synechocystis PCC 6803 following nitrogen starvation [72]. Although these results still require genetic confirmation, they do point out a much broader role of NtcA in the control of $\mathrm{C} / \mathrm{N}$ metabolic balance than that summarized in Fig. 4. In a regulatory perspective, by switching the early steps in $\mathrm{C} / \mathrm{N}$ metabolism on and off according to the availability of one nutrient relative to another, cyanobacterial cells ensure rapid responses to environmental changes, so that the $\mathrm{C} / \mathrm{N}$ balance can be maintained while saving resources that would have to be spent to control later metabolic steps. In principle, 2-OG could regulate the $\mathrm{C} / \mathrm{N}$ balance alone, as it 
controls the early steps of both carbon and nitrogen acquisition through its action on NdhR and NtcA (Fig. 4). Why then do cyanobacterial cells require multiple signals to control the $\mathrm{C} / \mathrm{N}$ metabolic balance? The answer may be linked to the response efficiency and the sensitivity of the control system. While the intracellular concentration of 2-OG, which acts as a carbon skeleton for nitrogen assimilation, may change rapidly following shifts in nitrogen availability, its variation may be slower and of a smaller amplitude following changes in carbon availability because the Krebs cycle is situated far downstream of inorganic-carbon assimilation steps (Fig. 1; Fig. 3). By also using 2-PG, whose levels are directly dependent on carbon availability through the activity of Rubisco, cyanobacterial cells can ensure a proper and timely balance of $\mathrm{C} / \mathrm{N}$ metabolism.

In the proposed model, we concentrated on 2-PG and 2-OG as signals, and NtcA and NdhR as transcription factors, because these elements are the most thoroughly studied so far using biochemical, genetic and structural approaches. Other metabolites involved in carbon fixation or photorespiration are also potential signals for carbon metabolism, and other regulatory proteins may also exist [4]. For example, additional robustness in the regulatory systems may be afforded by linkage with cAMP levels as discussed above, although the evidence for such linkages are, for cyanobacteria, largely based upon circumstantial evidence and remain to be fully validated experimentally. In addition, the role of small, non-coding, regulatory RNA in controlling cyanobacterial gene expression is only beginning to be appreciated and, potentially, these could also influence the regulation of $\mathrm{C} / \mathrm{N}$ balance. These include the recent discovery of a glutamine-responsive riboswitch that enables fast regulation of glutamine synthase, the key enzyme in the 2-OG utilizing GS-GOGAT cycle [5]. In the future, it will also be interesting to study how the 2-PG and 2-OG signaling pathways are modulated by, or respond to, environmental factors other than carbon and nitrogen availability, such as

422 light/dark transition, and light quantity or quality. Other nutritional, developmental, or environmental factors may also shape $\mathrm{C} / \mathrm{N}$ balance and the underlying signaling pathways. As an illustration to this idea, the $\mathrm{C} / \mathrm{N}$ ratio differs in vegetative cells and heterocysts in Anabaena PCC 7120, with heterocysts accumulating a higher level of nitrogen reserves, consistent with their dedicated function of $\mathrm{N}_{2}$ fixation for the whole filaments [73].

Cyanobacteria are a diverse group of prokaryotes. Marine cyanobacteria from oligotrophic oceans live in a much stable environment, with less nutritional variations than those from freshwater habitats. Consequently, many of these marine strains such as 
Prochlorococcus species have evolved streamlined genomes, including the loss of many regulatory features [74]. Similarly, the interaction between NtcA and its target DNA in these strains become much less responsive to 2-OG, as a consequence of their long-term adaptation to the rather stable environment $[75,76]$.

\section{Tools used for and learned from the study of 2-OG and 2-PG signaling}

The study of 2-OG and 2-PG signaling benefited and will continue to benefit from various tools established in the past. For example, a series of 2-OG analogs have been designed and synthesized for investigating the signaling role of 2-OG [29,37,39,40] (Fig. 2). In addition to the nonmetabolizable analog DFPA (described above), which gives a F-NMR signal with little background in living organisms, other 2-OG analogs include those that can mimic either the ketone form or the ketal form of 2-OG [37], which exist in equilibrium in living organisms. It was found that 2-MPA (Fig. 2a), a mimic of the ketone form of 2-OG, can play the same signaling role as 2-OG in the cyanobacterium Anabaena PCC 7120, while other analogs corresponding to mimics of the ketal form of 2-OG were unable to do so [37]. Attempts were also made to develop an affinity column to enrich or purify 2-OG receptors and binding proteins [77]. To that end, the structure-activity relationship of 2-OG signaling has been explored in order to determine to what extent 2-OG can be modified without affecting its signaling functions [37-40,77]. Two analogs, DFMPA and DMPA, obtained after modification of 2-OG at the C2 and C4 positions at the same time (Fig. 2a), still retained a signaling function in Anabaena PCC 7120, like 2-OG [38,39]. Based on this information, DMPA was conjugated to a solid resin by click chemistry, and the affinity resin obtained was used to purify the 2-OG receptor NtcA, which was overexpressed in E. coli [77]. Given the fact that 2-OG binding proteins, such as PII, NtcA abd NdhR, do not share a common motif for 2-OG recognition $[43,46,50,56]$, different analogs of $2-O G$ may be conjugated to a solid resin for identification of new 2-OG binding proteins or receptors.

When 2-OG or its analogs are used, one problem is how to get them into the cells so that the intracellular pool of 2-OG can be manipulated. So far, two methods have proved successful. The first relies on the expression of the $k g t P$ gene from E. coli, which encodes a permease for C5-compounds including 2-OG $[29,58,78]$. The second is the use of the membrane-permeable analog of 2-OG, dmOG (Fig. 2a), which is commercially available. Under the action of esterases, dmOG is converted to 2-OG in vivo, and hence the level of 2-OG can 
462

463

464

465

466

467

468

469

470

471

472

473

474

475

476

477

478

479

480

481

482

483

484

485

486

487

488

489

490

491

492

493

be increased within the cells of either E. coli or Synechocystis PCC 6803 after treatment with dmOG $[30,46]$.

Changes in the 2-OG pool in cells may be fast and may vary on an individual cell basis according to cell age or cell cycle progression. Thus, it is interesting to measure the 2-OG level and follow its changes in live single cells, and in real time. Several 2-OG biosensors based on the technique of FRET (fluorescence resonance energy transfer) have been developed [7982]. These 2-OG biosensors rely on either 2-OG-dependent protein-protein interaction or protein conformational changes induced by 2-OG binding. At least in vitro, FRET efficiency of these 2-OG biosensors is quantitatively correlated to 2-OG concentrations.

Compared to the variety of tools available for studies on 2-OG signaling, far fewer have been developed for 2-PG. A 2-PG transporter system has been described in Arabidopsis thaliana [83], which may be used to increase the 2-PG pool in the cells. Overexpression of a gene encoding a phosphoglycolate phosphatase was proven to be successful for decreasing the internal pool of 2-PG in cyanobacteria $[46,47]$.

A number of studies, including those reviewed here, revealed that 2-OG is not only an essential metabolite for biosynthesis, but also a signaling molecule in bacteria, plants, animals and humans $[2,25,29-31,43,46]$. Therefore, tools developed to study 2-OG signaling are of considerable interest to the scientific community in general.

\section{Concluding remarks and future perspectives}

Cyanobacteria are the ancestors of plastids, and they thus have much in common with algae and higher plants in terms of $\mathrm{C} / \mathrm{N}$ metabolic coupling. Although the sensors/receptors involved may differ, the signalling nature of $2-O G$ and $2-P G$ is very likely a common feature in photosynthetic organisms. The signalling function of 2-OG is already well established in bacteria, plants, animals and humans, although the detailed mechanism in most cases remains poorly understood, except the conservation of the PII pathway in bacteria and plants, although distinct features have evolved for PII in algae and plants [2,35]. Several metabolites and amino acids affect nitrogen metabolism in plants, but it remains unclear whether they are directly involved in signalling in nitrogen control [84]. The signal for carbon limitation in plants is also not clear, but 2-PG and other metabolites from photorespiration and the CBB cycle are the best candidates in light of the current studies. Indeed, it has been shown in Arabidopsis thaliana that a pglp1 mutant defective in the gene encoding 2-PG phosphatase accumulates 
a high level of 2-PG but much less starch than the wild type [85]. These results are consistent with a possible role of 2-PG in signalling a carbon limitation. It is also reported recently that the Krebs cycle display a strikingly coordinated regulation upon nitrogen starvation in the diatom Phaeodactylum tricornutum, and this control is mediated through a conserved transcription factor bZIP14 [86]. These data clearly establish a certain parallel between eukaryotic algae and cyanobacteria in terms of nitrogen control. It would therefore be interesting to identify the receptors and the corresponding signalling pathways in plants. The concepts developed so far based on studies in cyanobacteria, and the various tools that are available, could help to stimulate research in this direction in plants.

As we commented in this review, increasing numbers of studies have provided evidence that photorespiration and the Krebs cycle are not only important metabolic pathways but also critical players in signalling in $\mathrm{C} / \mathrm{N}$ metabolic control. Understanding the underlying mechanism of $\mathrm{C} / \mathrm{N}$ balance is important not only for fundamental research but also for applied research. For example, bioenergy research largely consists of shifting the $\mathrm{C} / \mathrm{N}$ balance towards the production of carbon-storage compounds for use as biofuel, and understanding different control points in $\mathrm{C} / \mathrm{N}$ balance could therefore be instrumental in increasing the efficiency of biofuel production. Better understanding of the $\mathrm{C} / \mathrm{N}$ balance could also be instructive for improving crop production, because properly engineering the control points of $\mathrm{C} / \mathrm{N}$ balance could optimize the efficiency of the uptake, assimilation, and metabolism of carbon and nitrogen nutrients. Remarkably, a recent study demonstrates that two protein partners GRF4 and DELLA coordinate pant growth and C/N metabolism, and points to new breeding strategies for agriculture [87]. The broad scope of and the general interest in this field, combined with the essential function of $\mathrm{C} / \mathrm{N}$ metabolic control, will promote further investigations and allow us to answer challenging questions in the future.

\section{Acknowledgements}

520 Work related to the topics in the authors' laboratories are supported by the Key Research 521 Program of Frontier Sciences of the Chinese Academy of Sciences Grant QYZDJ-SSW-SMC016, 522 the National Natural Science Foundation of China (www.nsfc.gov.cn) Grants 31630001, and 523 the Strategic Priority Research Program of the Chinese Academy of Sciences Grant 524 XDB08020300 and United States Department of Energy (DOE), Office of Science, Basic 
525 Energy Sciences, grant no. DE-FG02-08ER15968. We thank Isabel Hanson for English 526 corrections.

527

\section{References}

5291 Giordano, M. (2013) Homeostasis: An underestimated focal point of ecology and

$530 \quad$ evolution. Plant Science 211, 92-101

5312 Chellamuthu, V.R. et al. (2013) From cyanobacteria to plants: Conservation of PII 532 functions during plastid evolution. Planta 237, 451-462

5333 Herrero, A. et al. (2004) Cellular differentiation and the NtcA transcription factor in 534 filamentous cyanobacteria. FEMS Microbiol. Rev. 28, 469-487

5354 Burnap, R. et al. (2015) Regulation of CO2 Concentrating Mechanism in

$536 \quad$ Cyanobacteria. Life 5, 348-371

$5375 \quad$ Klähn, S. et al. (2018) A glutamine riboswitch is a key element for the regulation of 538 glutamine synthetase in cyanobacteria. Nucleic Acids Res. DOI: 10.1093/nar/gky709

5396 Du, J. et al. (2014) Characterisation of cyanobacterial bicarbonate transporters in $E$. 540 coli shows that SbtA homologs are functional in this heterologous expression system. $541 \quad$ PLoS One 9, e115905

5427 Selim, K.A. et al. (2018) P॥l-like signaling protein SbtB links cAMP sensing with cyanobacterial inorganic carbon response. Proc. Natl. Acad. Sci. U. S. A. 115, 48614869

5458 Ponce-Toledo, R.I. et al. (2017) An early-branching freshwater cyanobacterium at the origin of plastids. Curr. Biol. 27, 386-391

5479 Rippka, R. et al. (1979) Generic assignments, strain histories and properties of pure cultures of cyanobacteria. J. Gen. Microbiol. 111, 1-61

54910 Zhang, C.C. et al. (1989) Molecular and genetical analysis of the fructose-glucose 550 transport system in the cyanobacterium Synechocystis PCC6803. Mol Microbiol 3, 1221-1229

55211 Bauwe, H. et al. (2010) Photorespiration: players, partners and origin. Trends Plant Sci.

$553 \quad 15,330-336$

55412 Moroney, J. V. et al. (2013) Photorespiration and carbon concentrating mechanisms: Two adaptations to high $\mathrm{O}_{2}$, low $\mathrm{CO}_{2}$ conditions. Photosynth. Res. 117, 121-131

55613 Hagemann, M. and Bauwe, H. (2016) Photorespiration and the potential to improve 
55814 Turmo, A. et al. (2017) Carboxysomes: metabolic modules for $\mathrm{CO}_{2}$ fixation. FEMS Microbiol. Lett. 364, fnx176

56015 Price, G.D. et al. (2013) The cyanobacterial CCM as a source of genes for improving photosynthetic $\mathrm{CO}_{2}$ fixation in crop species. J. Exp. Bot. 64, 753-768

562

Young, J.D. et al. (2011) Mapping photoautotrophic metabolism with isotopically non

563 stationary ${ }^{13} \mathrm{C}$ flux analysis. Metab. Eng. 13, 656-665

Xiong W. et al. (2015) The plasticity of cyanobacterial metabolism supports direct $\mathrm{CO}_{2}$ conversion to ethylene. Nat. Plants 1, 15053

566

Zhang, C.C. et al. (2006) Heterocyst differentiation and pattern formation in cyanobacteria: A chorus of signals. Mol. Microbiol. 59, 367-375

568

Herrero, A. et al. (2016) The multicellular nature of filamentous heterocyst-forming cyanobacteria. FEMS Microbiol Rev 40, 831-854

Schneegurt, M.A. et al. (2000) Metabolic rhythms of a diazotrophic cyanobacterium, Cyanothece sp. strain ATCC 51142, heterotrophically grown in continuous dark. J. Phycol. 36, 107-117

57321 Muro-Pastor, M.I. et al. (2005) Ammonium assimilation in cyanobacteria. Photosynth. Res. 83, 135-150

Ohashi, Y. et al. (2011) Regulation of nitrate assimilation in cyanobacteria. J. Exp. Bot.

$$
62,1411-1424
$$

Shih, P.M. et al. (2013) Improving the coverage of the cyanobacterial phylum using diversity-driven genome sequencing. Proc. Natl. Acad. Sci. U. S. A. 110, 1053-1058

Reitzer, L. (2003) Nitrogen assimilation and global regulation in Escherichia coli. Annu. Rev. Microbiol. 57, 155-76

Huergo, L.F. and Dixon, R. (2015) The emergence of 2-oxoglutarate as a master regulator metabolite. Microbiol. Mol. Biol. Rev. 79, 419-435

26 Fulda, S. et al. (2006) Proteome analysis of salt stress response in the cyanobacterium Synechocystis sp. strain PCC 6803. Proteomics 6, 2733-2745

Stöckel, J. et al. (2011) Diurnal rhythms result in significant changes in the cellular protein complement in the cyanobacterium Cyanothece 51142. PLoS One 6, e16680 

heterocyst differentiation under repressive conditions in Anabaena sp. PCC 7120. Proc. Natl. Acad. Sci. U. S. A. 102, 9907-9912 Doucette, C.D. et al. (2011) a-ketoglutarate coordinates carbon and nitrogen utilization via enzyme I inhibition. Nat. Chem. Biol. 7, 894-901

594

31 He, W. et al. (2004) Citric acid cycle intermediates as ligands for orphan G-proteincoupled receptors. Nature 429, 188-193

Haas, R. et al. (2016) Intermediates of metabolism: From bystanders to signalling molecules. Trends Biochem. Sci. 41, 460-471

33 You, C. et al. (2013) Coordination of bacterial proteome with metabolism by cyclic AMP signalling. Nature 500, 301-306

600

Araujo, W.L. et al. (2014) 2-Oxoglutarate: linking TCA cycle function with amino acid, 601 glucosinolate, flavonoid, alkaloid, and gibberellin biosynthesis. Front. Plant Sci. 5, 552

602 Uhrig, R.G. et al. (2009) PII in higher plants: a modern role for an ancient protein. , 603 Trends Plant Sci. 14, 505-511

60436 Rabinowitz, J.D. and Silhavy, T.J. (2013) Systems biology: metabolite turns master 605 regulator. Nature 500, 283-4

60637 Chen, H. et al. (2006) Studying the signaling role of 2-oxoglutaric acid using analogs 607 that mimic the ketone and ketal forms of 2-oxoglutaric acid. Chem. Biol. 13, 849-856

60838 Liu, X. et al. (2013) Structural requirements of 2-oxoglutaric acid analogues to mimic 609 its signaling function. Org. Lett. 15, 4662-4665

61039 Liu, X. et al. (2011) 2-Difluoromethylene-4-methylenepentanoic acid, a paradoxical 611 probe able to mimic the signaling role of 2-oxoglutaric acid in cyanobacteria. Org. Lett. $612 \quad 13,2924-2927$

61340 Wang, Y. et al. (2014) Mimicking the 2-oxoglutaric acid signalling function using 614 molecular probes: insights from structural and functional investigations. Org. Biomol. $615 \quad$ Chem. 12, 4723-9

61641 Ziarelli, F. et al. (2012) High resolution magic angle spinning NMR to investigate 617 ligand-receptor binding events for mass-limited samples in liquids. J. Pharm. Biomed. 618 Anal. 59, 13-17

61942 Chen, H. et al. (2013) ${ }^{19}$ F NMR: a valuable tool for studying biological events. Chem. 620 Soc. Rev. 42, 7971 
62143 Zhao, M.-X. et al. (2010) Structural basis for the allosteric control of the global

622 transcription factor NtcA by the nitrogen starvation signal 2-oxoglutarate. Proc. Natl.

$623 \quad$ Acad. Sci. U. S. A. 107, 12487-92

62444 Klähn, S. et al. (2015) Integrated transcriptomic and metabolomic characterization of 625 the low-carbon response using an ndhR mutant of Synechocystis sp. PCC 6803. Plant

$626 \quad$ Physiol. 169, 1787-1806

62745 Wang, H.L. et al. (2004) Alterations in global patterns of gene expression in 628 Synechocystis sp. PCC 6803 in response to inorganic carbon limitation and the 629 inactivation of $n d h R$, a LysR Family Regulator. J. Biol. Chem. 279, 5739-5751

63046 Jiang, Y.-L. et al. (2018) Coordinating carbon and nitrogen metabolic signaling through 631 the cyanobacterial global repressor NdhR. Proc. Natl. Acad. Sci. U. S. A. 115, 403-408

63247 Haimovich-Dayan, M. et al. (2015) Does 2-phosphoglycolate serve as an internal signal 633 molecule of inorganic carbon deprivation in the cyanobacterium Synechocystis sp. PCC 634 6803? Environ. Microbiol. 17, 1794-1804

63548 Daley, S.M.E. et al. (2012) Regulation of the cyanobacterial CO2-concentrating 636 mechanism involves internal sensing of NADP+ and $\alpha$-ketogutarate levels by transcription factor CcmR. PLoS One 7, e41286

63849 Huergo, L.F. et al. PII signal transduction proteins: Nitrogen regulation and beyond. , 639 FEMS Microbiology Reviews, 37. (2013) , 251-283

64050 Fokina, O. et al. (2010) Mechanism of 2-oxoglutarate signaling by the Synechococcus elongatus PII signal transduction protein. Proc. Natl. Acad. Sci. U. S. A. 107, 1976019765

64351 Espinosa, J. et al. (2014) PipX, the coactivator of NtcA, is a global regulator in $644 \quad$ cyanobacteria. Proc. Natl. Acad. Sci. U. S. A. 111, E2423-E2430

64552 Llácer, J.L. et al. (2007) The crystal structure of the complex of PII and acetylglutamate 646 kinase reveals how PIl controls the storage of nitrogen as arginine. Proc. Natl. Acad. Sci. U. S. A. 104, 17644-17649

64853 Maheswaran, M. et al. (2004) Complex formation and catalytic activation by the PII 649 signaling protein of $\mathrm{N}$-acetyl-L-glutamate kinase from Synechococcus elongatus strain $650 \quad$ PCC 7942. J. Biol. Chem. 279, 55202-55210

65154 Espinosa, J. et al. (2006) Interaction network in cyanobacterial nitrogen regulation: PipX, a protein that interacts in a 2-oxoglutarate dependent manner with PII and 
653

$654 \quad 55$

655

656

$657 \quad 56$

658

659

660

661

662

663

664

665

666

667

668

669

670

671

672

673

674

675

676

677

678

679

680

681

682

683

684

NtcA. Mol. Microbiol. 61, 457-469

55 Wheatley, N.M. et al. (2016) A PII-Like Protein Regulated by Bicarbonate: Structural and Biochemical Studies of the Carboxysome-Associated CPII Protein. J. Mol. Biol. 428, 4013-4030

Llácer, J.L. et al. (2010) Structural basis for the regulation of NtcA-dependent transcription by proteins PipX and PII. Proc. Natl. Acad. Sci. U. S. A. 107, 15397-15402

57 Tanigawa, R. et al. (2002) Transcriptional activation of NtcA-dependent promoters of Synechococcus sp. PCC 7942 by 2-oxoglutarate in vitro. Proc. Natl. Acad. Sci. U. S. A. 99, 4251-4255

58 Li, J.H. et al. (2003) An increase in the level of 2-oxoglutarate promotes heterocyst development in the cyanobacterium Anabaena sp. strain PCC 7120. Microbiology 149, 3257-3263

59 Eisenhut, M. et al. (2008) The photorespiratory glycolate metabolism is essential for cyanobacteria and might have been conveyed endosymbiontically to plants. Proc. Natl. Acad. Sci. U. S. A. 105, 17199-204

60 Takahashi, Y. et al. (2004) Roles of CmpR, a LysR family transcriptional regulator, in acclimation of the cyanobacterium Synechococcus sp. strain PCC 7942 to low- $\mathrm{CO}_{2}$ and high-light conditions. Mol. Microbiol. 52, 837-845

61 Lieman-Hurwitz, J. et al. (2009) A cyanobacterial AbrB-like protein affects the apparent photosynthetic affinity for $\mathrm{CO}_{2}$ by modulating low- $\mathrm{CO}_{2}$-induced gene expression. Environ. Microbiol. 11, 927-936

62 Picossi, S. et al. (2015) The LysR-type transcription factor PacR is a global regulator of photosynthetic carbon assimilation in Anabaena. Environ. Microbiol. 17, 3341-3351

63 Nishimura, T. et al. (2008) Mechanism of low $\mathrm{CO}_{2}$-induced activation of the $\mathrm{cmp}$ bicarbonate transporter operon by a LysR family protein in the cyanobacterium Synechococcus elongatus strain PCC 7942. Mol. Microbiol. 68, 98-109

64 Figge, R.M. et al. (2001) Characterization and analysis of an NAD(P)H dehydrogenase transcriptional regulator critical for the survival of cyanobacteria facing inorganic carbon starvation and osmotic stress. Mol. Microbiol. 39, 455-468

65 Woodger, F.J. et al. (2007) Transcriptional regulation of the $\mathrm{CO}_{2}$-concentrating mechanism in a euryhaline, coastal marine cyanobacteriurn, Synechococcus sp. strain PCC 7002: Role of NdhR/CcmR. J. Bacteriol. 189, 3335-3347 
68566 Hackenberg, C. et al. (2012) Low-carbon acclimation in carboxysome-less and 686 photorespiratory mutants of the cyanobacterium Synechocystis sp. strain PCC 6803.

$687 \quad$ Microbiology 158, 398-413

68867 Stanier, R.Y. and Cohen-Bazire, G. (1977) Phototrophic Prokaryotes: The 689 Cyanobacteria. Annu. Rev. Microbiol. 31, 225-274

69068 Conrad, M. et al. (2014) Nutrient sensing and signaling in the yeast Saccharomyces $691 \quad$ cerevisiae. FEMS Microbiol. Rev. 38, 254-299

69269 Berridge, M.J. et al. (2003) Calcium signalling: Dynamics, homeostasis and 693 remodelling. Nature Rev. Mol. Cell Biol. 4, 517-529

69470 Kaplan, A. and Reinhold, L. (1999) $\mathrm{CO}_{2}$ concentrating mechanisms in microorganisms. $695 \quad$ Transport 50, 539-570

69671 Lopez-Igual, R. et al. (2012) N and C control of ABC-type bicarbonate transporter Cmp 697 and its LysR-type transcriptional regulator $\mathrm{CmpR}$ in a heterocyst-forming 698 cyanobacterium, Anabaena sp. Environ. Microbiol. 14, 1035-1048

69972 Giner-Lamia, J. et al. (2017) Identification of the direct regulon of NtcA during early 700 acclimation to nitrogen starvation in the cyanobacterium Synechocystis sp. PCC 6803.

701 Nucleic Acids Res. 45, 11800-11820

70273 Teramoto, T. et al. (2018) Soft X-ray imaging of cellular carbon and nitrogen 703 distributions in heterocystous cyanobacterium. Plant Physiol. DOI:

704 10.1104/pp.17.01767

705

74 Scanlan, D.J. et al. (2009) Ecological Genomics of Marine Picocyanobacteria. Microbiol. 706 Mol. Biol. Rev. 73, 249-299

707

Domínguez-Martín, M.A. et al. (2018) Differential NtcA responsiveness to 2-

708 oxoglutarate underlies the diversity of $\mathrm{C} / \mathrm{N}$ balance regulation in Prochlorococcus. Front. Microbiol. 8, 1-16

Domínguez-Martín, M.A. et al. (2018) Distinct features of $\mathrm{C} / \mathrm{N}$ balance regulation in Prochlorococcus sp. strain MIT9313. FEMS Microbiol. Lett. 365, fnx278 Wang, Y. et al. (2014) A "click" chemistry constructed affinity system for 2-oxoglutaric acid receptors and binding proteins. Org. Biomol. Chem. 12, 6470-6475

71478 Seol, W. and Shatkin, A.J. (1991) Escherichia coli kgtP encodes an $\alpha$-ketoglutarate transporter. Proc. Natl. Acad. Sci. U. S. A. 88, 3802-3806 
of PII and PipX proteins provides a robust and specific biosensor for 2-oxoglutarate, a central metabolite and a signalling molecule. FEBS J. 281, 1241-1255

Zhang, C. et al. (2013) Quantitative monitoring of 2-oxoglutarate in Escherichia coli cells by a fluorescence resonance energy transfer-based biosensor. Appl. Microbiol. Biotechnol. 97, 8307-8316

81 Lüddecke, J. et al. (2017) PII protein-derived FRET sensors for quantification and livecell imaging of 2-oxoglutarate. Sci. Rep. 7, 1-13

724

82 Lüddecke, J. and Forchhammer, K. (2013) From PII signaling to metabolite sensing: A novel 2-oxoglutarate sensor that details PII - NAGK complex formation. PLoS One 8, 111

83 South, P.F. et al. (2017) Bile acid sodium symporter BASS6 can transport glycolate and is involved in photorespiratory metabolism in Arabidopsis thaliana. Plant Cell 29, 808823

84 Gent, L. and Forde, B.G. (2017) How do plants sense their nitrogen status? J. Exp. Bot. $68,2531-2540$

85 Timm, S. et al. (2012) High-to-low $\mathrm{CO}_{2}$ acclimation reveals plasticity of the photorespiratory pathway and indicates regulatory links to cellular metabolism of Arabidopsis. PLoS One 7, e42809

86 Matthijs, M. et al. (2017) The transcription factor bZIP14 regulates the TCA cycle in the diatom Phaeodactylum tricornutum. EMBO J. 61, 316-324

87 Li, S. et al. (2018) Modulating plant growth - metabolism coordination for sustainable agriculture. Nature 560, 595-560

739

\section{Figure legends}

Fig. 1. Coupling between carbon and nitrogen metabolism. Inorganic carbon, as $\mathrm{CO}_{2}$ or $\mathrm{HCO}_{3}{ }^{-}$ , enters the cells and it is in the form of $\mathrm{CO}_{2}$ that is assimilated into organic carbons through the $\mathrm{CBB}$ cycle. Note that the uptake of $\mathrm{CO}_{2}$ is energetically driven by specialized forms of the $\mathrm{NDH}-1$ complex in thylakoid membranes and results in the formation of $\mathrm{HCO}_{3}{ }^{-}$which is transported into Rubisco-containing carboxysomes before being reconverted to $\mathrm{CO}_{2}$ (see text for details). Fixed carbon through the CBB cycle is further used for biosynthesis or to feed the Krebs cycle (incomplete in cyanobacteria in general due to the lack of the 2-OG 
749

750

751

752

753

754

755

756

757

758

759

760

761

762

763

764

765

766

767

768

769

770

771

772

773

774

775

776

777

778

779

780

dehydrogenase), which produces intermediates for a variety of carbon compounds including lipids. Different forms of inorganic nitrogen can be used by cyanobacteria, and are assimilated in the form of $\mathrm{NH}_{4}{ }^{+}$through the GS-GOGAT cycle using 2-OG as a carbon skeleton. Glu and GIn, the two amino acids produced from the GS-GOGAT cycle are also important nitrogen donors for the synthesis of a variety of nitrogen-containing compounds.

Fig. 2. (a) 2-OG and its analogs that can be used for the study of 2-OG regulation. 2-OG, 2oxoglutarate; dmOG, dimethyl 2-oxoglutarate, a membrane permeable analog of 2-OG; DFPA, a fluorinated nonmetabolisable analog of 2-OG, which can be traced in vivo by ${ }^{19} \mathrm{~F}-\mathrm{NMR}$; 2 MPA, a mimic of the ketone form of 2-OG; DFMPA, a hybrid of DFPA and 2-MPA; and DMPA, an analog of 2-OG that can be conjugated to resins for enrichment of 2-OG-binding proteins. (b) Schematic representation on the signalling mechanism of NtcA based on structural studies. NtcA is a homodimer and a transcriptional activator, each subunit containing an effectorbinding domain (EBD) and a DNA-binding domain (DBD). The two helices in the DBDs (in green) interact with target DNA. When 2-OG binds to NtcA at the EBDs, structural conformation changes are induced, shortening the distance between the two DNA-binding helices from 37 to $34 \AA$, optimal for interaction with DNA and thus transcriptional control.

Fig. 3. Mechanism of carbon metabolic control.

(a) Outline of the transcriptional control. The activator $\mathrm{CmpR}$ and the repressor $\mathrm{NdhR}$ are the two major transcriptional factors for the regulation of $\mathrm{CCM}\left(\mathrm{CO}_{2}\right.$ concentrating mechanism). $\mathrm{CmpR}$ controls the $\mathrm{cmp}$ operon, encoding a high affinity bicarbonate transport system, whereas NdhR represses several inorganic carbon uptake systems, including Ndh-13, a highaffinity $\mathrm{CO}_{2}$ uptake system; SbtA, a high-affinity bicarbonate transport system; and BicA, a low affinity bicarbonate transport system. The signal that regulates $\mathrm{CmpR}$ is not yet fully understood. NdhR is under the dual control of 2-PG and 2-OG, acting as an anti-repressor and co-repressor, respectively. When $\mathrm{CO}_{2}$ is limiting, 2-PG is produced by the oxygenase activity of Rubisco and binds to NdhR. The 2-PG/NdhR complex is unable to fix to DNA, thus the CCMrelated operon is expressed, leading to enhanced $\mathrm{CO}_{2}$ /bicarbonate uptake. If $\mathrm{CO}_{2}$ level is sufficient, the carboxylase activity of Rubisco is favored, leading to $\mathrm{CO}_{2}$ fixation. Under such a condition, the 2-PG level is low, while that of 2-OG is high, leading to the formation of the 2OG/NdhR complex and a stronger repression of the CCM-related gene expression. 
781 (b) Mechanism of action of NdhR. NdhR is a tetramer and a repressor, with two compact 782 subunits (in cyan) and two extended subunits (in orange). Under nitrogen starvation, 2-OG 783 binds at the inter-domain cleft between the RDs (regulatory domain). In the 2-OG bound form, 784 the distance between the two helices at the corresponding DBDs (DNA-binding domains) is 29 $785 \AA$ A , favorable for DNA binding, thus transcriptional repression. Under a carbon starvation, 2-PG 786 binds at the intra-domain cleft of the RDs, leading to shortening of the distance between the 787 two DNA-binding helices to $25 \AA ̊$, unfavorable for DNA binding, thus relieving transcriptional 788 repression.

789

790 Fig. 4. A model on the mechanism of $\mathrm{C} / \mathrm{N}$ metabolic balance in cyanobacteria.

791 (a) Under nitrogen limitation or carbon oversupply, the 2-OG level increases while that of 2792 PG decreases. The 2-OG/NtcA complex activates genes involved in nitrogen uptake and 793 assimilation, while the 2-OG/NdhR complex represses the $\mathrm{CCM}\left(\mathrm{CO}_{2}\right.$ concentrating 794 mechanism)-related gene expression so that carbon uptake decreases. The joint action of the 795 two transcriptional complexes help the cells to regain $\mathrm{C} / \mathrm{N}$ balance.

796 (b) Under carbon limitation or nitrogen oversupply, the 2-OG level drops while that of 2-PG 797 increases. The apo form of NtcA has a much lower DNA binding activity, thus leading to a 798 decrease in nitrogen uptake and assimilation. Meanwhile, the 2-PG/NdhR complex cannot act 799 as a repressor, so that genes related to CCM is expressed. Consequently, the $\mathrm{C} / \mathrm{N}$ balance is 800 achieved. 


\section{Outstanding questions for future research}

- The key enzyme for autotrophic growth, Rubisco (ribulose 1,5-bisphosphate carboxylase/oxygenase), is a bifunctional enzyme. What are the regulatory factors that allow cells to switch between $\mathrm{CO}_{2}$ fixation, catalyzed by the carboxylase activity, or photorespiration, catalyzed by the oxygenase activity?

- How do the 2-phosphoglycolate (2-PG) and 2-oxoglutarate (2-OG) signaling pathways, and hence the $\mathrm{C} / \mathrm{N}$ balance, respond to changes other than $\mathrm{C} / \mathrm{N}$ availability, such as light intensity?

- Are there other signals involved in $\mathrm{C} / \mathrm{N}$ balance in cyanobacteria? If there are, how are different signals integrated to coordinate $\mathrm{C} / \mathrm{N}$ metabolic balance?

- To what extent are changes in 2-PG and 2-OG levels correlated in cells? How do 2-PG and 2-OG signaling affect other regulations required for $\mathrm{C} / \mathrm{N}$ balance such as redirection of metabolic fluxes or enzymatic activities?

- To what extent are the signaling mechanisms identified from cyanobacteria conserved in eukaryotic algae and plants? 


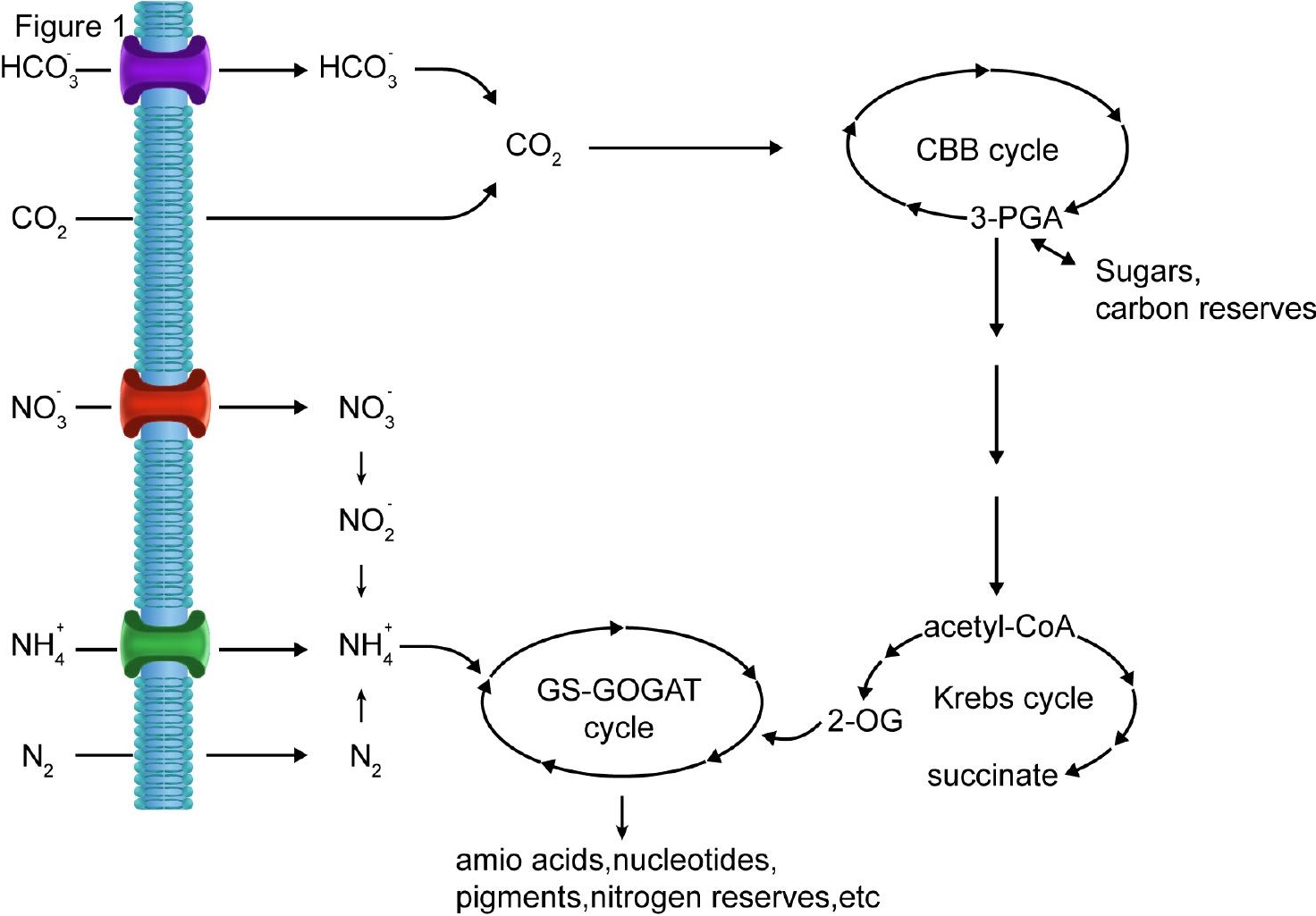




(-OGG

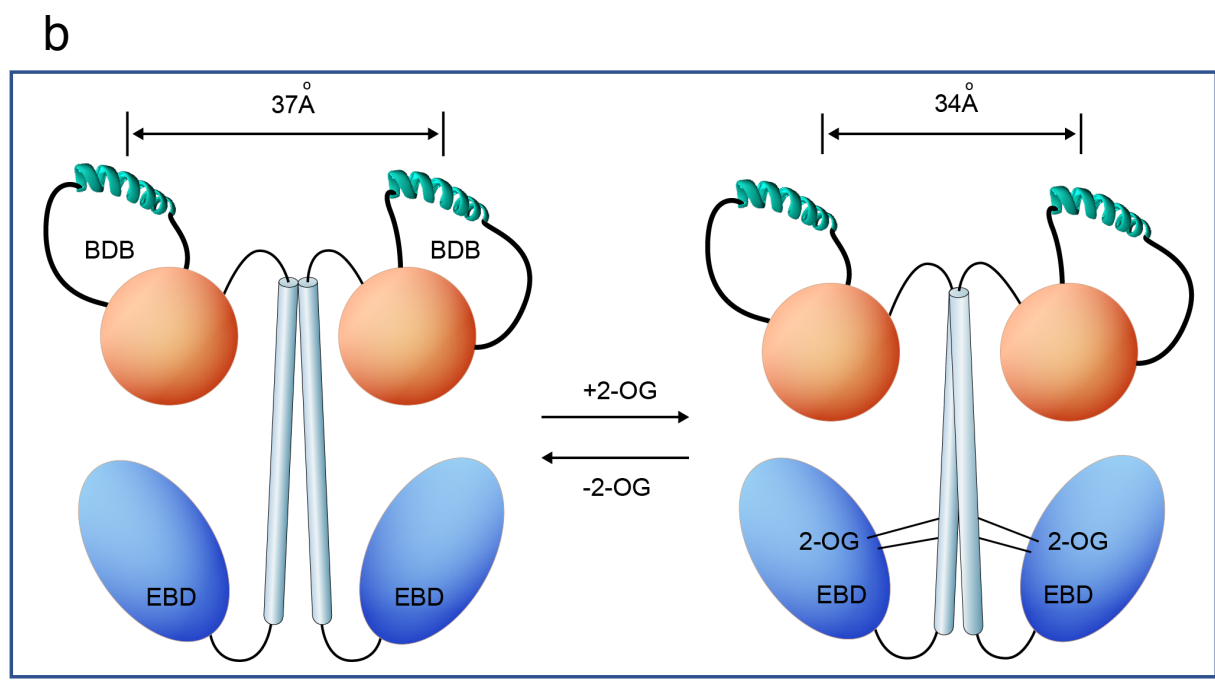


a

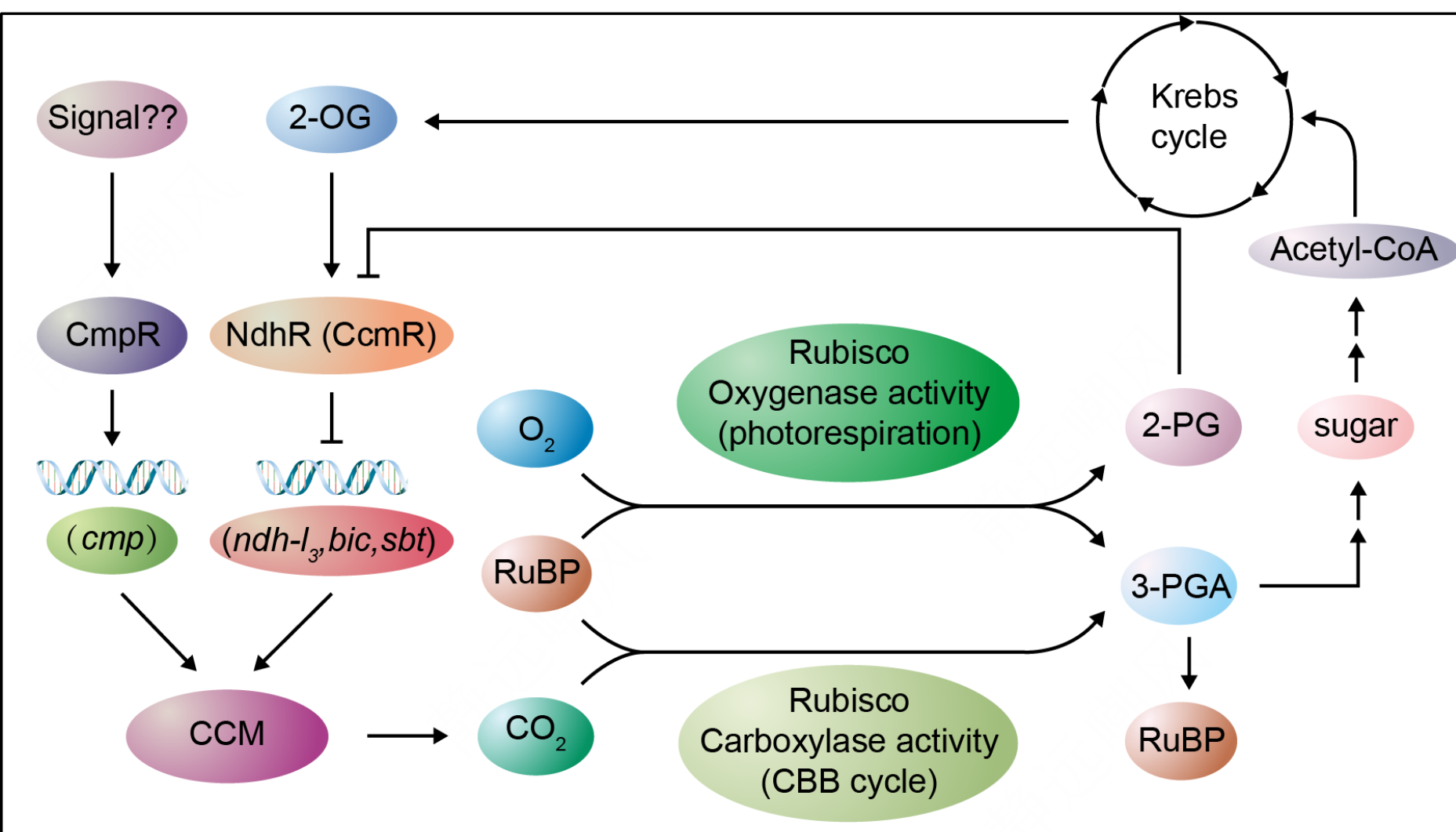

\section{b}

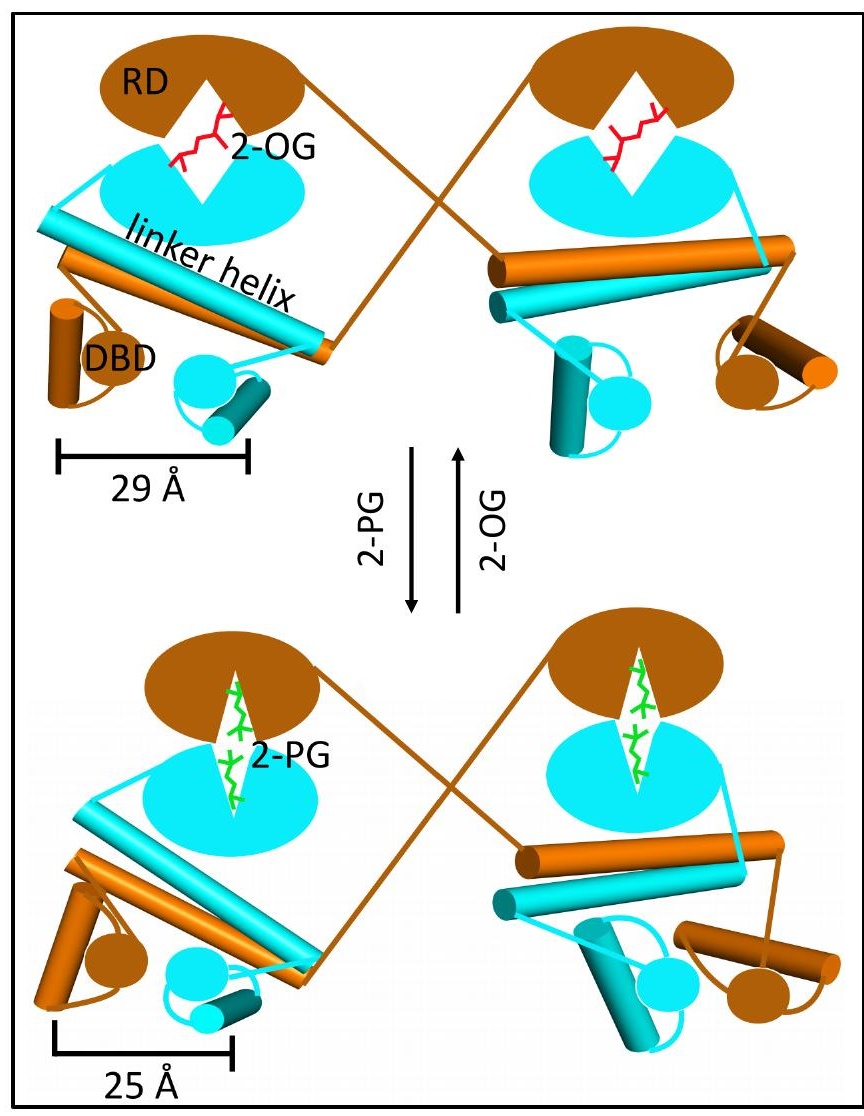



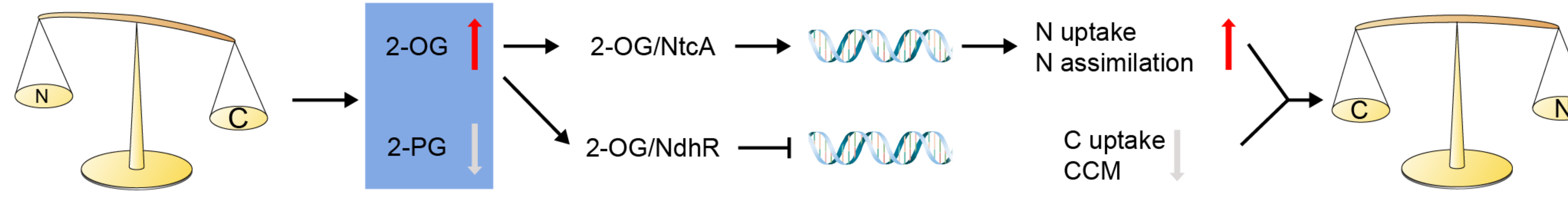

nitrogen limitation

(carbon oversupply)

b
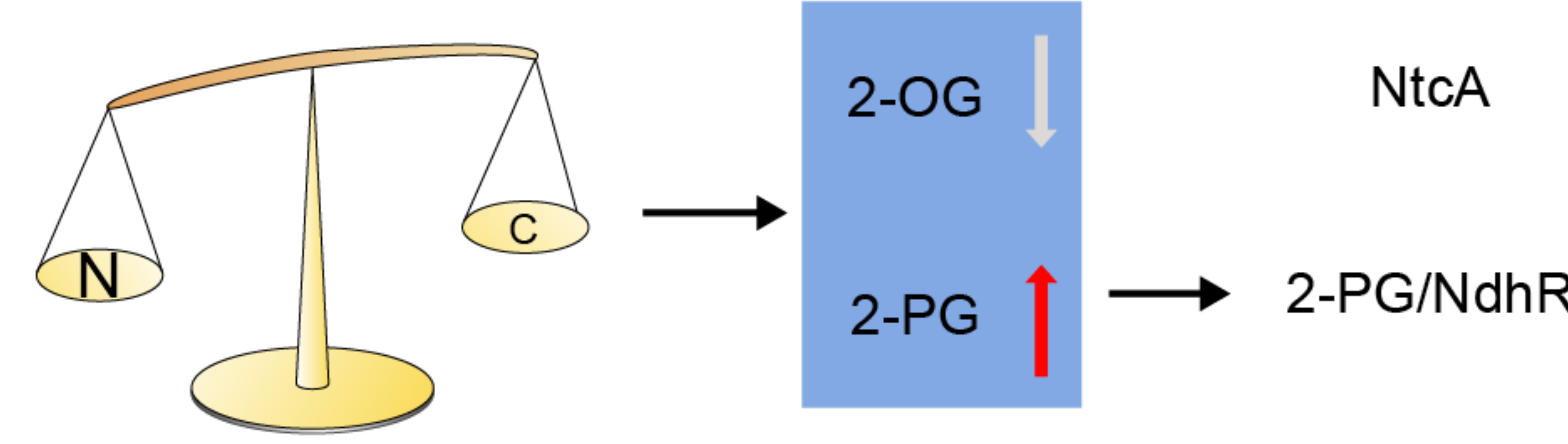

OONOO

$\mathrm{N}$ uptake

$\mathrm{N}$ assimilation

carbon limitation

(nitrogen oversupply) 